\title{
Alice in Wonderland: The effects of body size and movement on children's size perception and body representation in virtual reality
}

\begin{abstract}
Previous work shows that in adults, illusory embodiment of a virtual avatar can be induced using congruent visuomotor cues. Further, embodying different-sized avatars influences adults' perception of their environment's size. This study $(\mathrm{N}=92)$ investigated whether children are also susceptible to such embodiment and size illusions. Adults and 5-year-olds viewed a first-person perspective of different-sized avatars, moving either congruently or incongruently with their own body. Participants rated their feelings of embodiment over the avatar, as well as estimating the sizes of their body and objects in the environment. Unlike adults, children embodied the avatar regardless of visuomotor congruency. Both adults and children freely embodied different-sized avatars, and this affected their size perception in the surrounding virtual environment: in a small body they felt that objects were larger, and vice versa in a large body. Additionally, children felt that their body had grown in the large body condition. These findings have important implications for both our theoretical understanding of own-body representation, and our knowledge of perception in virtual environments.
\end{abstract}

Keywords: body, development, virtual reality, embodiment, perception

Funding: this work was supported by an Economic and Social Research Council-funded studentship and the Canadian Institute for Advanced Research (CIFAR) Azrieli Program in Mind, Brain, and Consciousness. 
Representing one's own body, as distinct from other objects in the environment (Bermúdez, 2005), is thought to result from sensory information about the body, including visual, tactile, and proprioceptive signals (Blanke \& Metzinger, 2009). The development of own-body representation is challenging, since children must 'keep track' of a body which is constantly changing and growing while their sensory systems also change substantially (De Klerk, Filippetti, \& Rigato, 2021). Because of this, it is proposed that children have increased plasticity in own-body representation (De Klerk et al., 2021; Dewe et al., 2021). Insights into this area are important not only from a theoretical standpoint, but also a practical one, as illusory embodiment of virtual bodies has potential applications in education (Hamilton, McKechnie, Edgerton, \& Wilson, 2021), entertainment, and therapy (Won et al., 2017).

\section{Bottom-up cues to body representation}

The foundations of adult-like body representation are laid down early, with lookingtime paradigms showing that infants as young as five months old are sensitive to cross-modal correlations between visual feedback and either their own movements, or felt touch (Bahrick \& Watson, 1985; M L Filippetti, Johnson, Lloyd-Fox, Dragovic, \& Farroni, 2013). Despite this sensitivity to multisensory correlations, it has long been suggested that explicit selfawareness only develops later, at around 18 months of age (Bischof-Köhler, 2012), when mirror self-recognition and personal pronouns emerge (Brooks-Gunn \& Lewis, 1984; Lewis \& Ramsay, 2004).

Beyond the first two years of life, children are better able to respond verbally regarding their experiences, and studies in the primary years suggest that there is substantial, experience-driven change in own-body representation at this age (De Klerk et al., 2021). This is often shown using bodily illusions where multisensory signals are manipulated to induce illusory embodiment over bodies or body parts other than one's own. (Here 'embodiment' is 
a common umbrella term which covers feelings of ownership or agency over a body; and the perceived position of one's body). The best-known bodily illusion is the rubber hand illusion (RHI; Botvinick \& Cohen, 1998) in which a fake hand is stroked at the same time and location as the participant's own, occluded hand. This congruency leads to embodiment of the fake hand. Likewise researchers evoke illusory ownership of a full body by using motion capture to give participants a first-person perspective of a virtual body which moves spatially and temporally congruently with their own (Peck, Seinfeld, Aglioti, \& Slater, 2013). These illusions can be conducted with body parts, or with a whole body in the full-body illusion (FBI). In adults, embodiment ratings in all versions are significantly higher after congruent experiences than after incongruent experiences, suggesting that visuotactile and visuomotor congruency are important cues to own-body representation in adults.

In children, these illusions likewise show that children between the ages of 4 and 13 years use multisensory correlations to determine ownership of a body part. In the RHI children experience an adult-like effect of visuotactile congruency on embodiment of a static hand (Cowie, Makin, \& Bremner, 2013; Cowie, Sterling, \& Bremner, 2016; Gottwald et al., 2021; Greenfield, Ropar, Smith, Carey, \& Newport, 2015) and an adult-like effect of visuomotor congruency on the embodiment of a moving hand (Dewe et al., 2021). However, children younger than 9 years also show very exaggerated shifts in perceived hand position towards the fake hand, regardless of congruency (Cowie et al., 2013, 2016; Filippetti \& Crucianelli, 2019; Gottwald et al., 2021); and some embodiment over non-human forms (Dewe et al., 2021). Therefore, visual capture is a particularly strong cue to embodiment for young children: the sight of the body in peripersonal space seems sufficient to evoke some embodiment of it, even when that is additionally regulated by multisensory congruency.

This is particularly pronounced in the case of whole-body ownership. During hand illusions, participants still have 'grounding' cues from the rest of the body; hands are also 
very prominent in the visual field from infancy (Fausey, Jayaraman, \& Smith, 2016). Therefore, and in contrast to the early effects of visuotactile congruency for hands, children tended to embody a displaced static full-body irrespective of congruency, the effects of which steadily increased from 6-11 years (Cowie, McKenna, Bremner, \& Aspell, 2017). When slightly older children (8-12 years) viewed a moving virtual body from a first-person perspective (Weijs, Macartney, Daum, \& Lenggenhager, 2021), visuomotor congruency did modulate ownership ratings - however, agency and affective responses were high in all conditions. Due to their different designs these studies are difficult to compare, but visual capture again emerges as a powerful factor in children's own-body representation - in these whole-body illusions, multisensory correlations only appear to regulate this effect later in the primary years.

This work suggests several important directions for the present study in terms of understanding children's bottom-up multisensory cues to own-body representation. First, it is important to further investigate visuomotor congruency in children's embodiment: there are few existing studies, with somewhat divergent results (Dewe et al., 2021; Weijs et al., 2021); moving bodies represent our most typical form of experience; and dynamic virtual embodied experiences are increasingly used in VR applications. Second, we need to understand how whole-body ownership in particular emerges in childhood, since existing data suggests it may be more malleable than hand ownership. Finally, existing work suggests that the use of multisensory cues for own-body representation is developing, but not yet adultlike, in the primary years. It is therefore important to establish how multisensory cues ground own-body representation in early childhood. We suggest 5 years as a sensible age to examine: this is younger than previous visuomotor studies, the youngest age at which verbal responses have been elicited from participants in bodily illusion studies (Cowie et al., 2013), and an age at 
which children respond well to fully-immersive VR (Negen, Heywood-Everett, Roome, \& Nardini, 2017).

\section{Top-down cues to body representation}

Bottom-up cues to embodiment are only part of the story of developing own-body representation. Adults also rely on top-down cues such as mental models of body form or size. For example, adults do not embody a wooden block, even with congruent visuotactile stimulation (Lenggenhager, Tadi, Metzinger, \& Blanke, 2007). Body size is a less well understood constraint to embodiment, and of particular interest in the context of bodily growth. The 'rubber band hypothesis' (de Vignemont, 2018) states that body size is a key constraint on embodiment, in particular that adults can embody a smaller, but not a larger, hand than their own (Marino, Stucchi, Nava, Haggard, \& Maravita, 2010; Pavani \& Zampini, 2007). Yet this literature is largely based on the hand alone. In contrast, adults have been shown to embody whole bodies which are either smaller or larger than their own (Banakou, Groten, \& Slater, 2013; van der Hoort, Guterstam, \& Ehrsson, 2011). This may result from experience of the whole body changing size throughout the lifespan. We argue that for identification of one's own full body, size may not constrain embodiment even in adults.

There is little data on how body size may constrain embodiment in children. Toddlers 'scale errors' - attempting to sit on a tiny doll's chair or in a toy car - are thought to result from an inability to inhibit automatic actions associated with certain objects and may not tell us much about early body size perception (Brownell, Zerwas, \& Ramani, 2007; DeLoache, Uttal, \& Rosengren, 2004). Using the RHI, Filippetti found that 6-8-year-olds were able to embody a larger hand than their own. However, this study did not provide the comparison case of smaller bodies. Likewise, using an augmented-reality MIRAGE box, Newport et al. (2015) showed that 8-15-year-olds felt that the illusion that their finger had been stretched. 
From this limited data our prediction is that children would be at least as willing as adults to accept bodies of different sizes, but we note the need to test this empirically.

When the relative size between one's body and environment changes, the observer can interpret this in one of two ways: either the environment has changed size and one's body is the same size, or the environment has stayed the same and one's body has changed. Previous investigations have found that participants tend towards the former: participants induced to inhabit a large body through visuotactile (van der Hoort et al., 2011) or visuomotor (Banakou et al. 2013) stimulation perceived objects in the environment as smaller, and vice versa. Importantly, participants anecdotally reported being unaware that the virtual body was a different size to their own, but instead felt surrounded by a differently sized world. It is important that, despite anecdotal and indirect evidence for this "bodyrelative scaling" (Linkenauger, Ramenzoni, \& Proffitt, 2010), to our knowledge there is little detail available from previous studies (Banakou et al., 2013; van der Hoort et al., 2011) on adults' subjective perception of own-body size during these experiences.

Likewise, no study has measured whether children engage in body-relative scaling during bodily illusions. Since young children encode their environment egocentrically instead of using external landmarks (Negen et al., 2017), one might predict that children's size perceptions would be affected by perceived body size to an even greater extent than those of adults. Further investigation into this area is needed.

\section{Present study}

Here we aimed to address outstanding questions regarding the bottom-up and topdown cues to own-body representation in childhood, across two experiments. Experiment 1 addressed the following three questions. Firstly, does full-body visuomotor congruency affect embodiment in young children (5 years old)? Secondly, does body size affect embodiment in 
children? Thirdly, does body size affect size perception in children? We predicted that children of this age would embody a virtual body regardless of visuomotor congruency or size; and that their size estimations would be more affected by perceived body size than those of adults. Experiment 2 was designed to directly address whether changes in body size lead to changes in the perceived size of the environment, or of the body itself. We predicted that adults would perceive changes in their environment but not their body; and that this effect would be magnified in children.

Across the two experiments, we used fully immersive virtual reality (VR) to manipulate the visuomotor congruency (Experiment 1) and body size (Experiments 1 \& 2) of a virtual body in 5-year-olds and adults. In Experiment 1, embodiment was assessed using a four-item questionnaire based on previous related studies (Cowie et al., 2018; Gottwald et al., 2021). The effect of body size on size perception was assessed by having participants estimate the size of unfamiliar and familiar objects before and after embodiment manipulations. In Experiment 1, participants estimated the sizes of unfamiliar objects manually, as in Banakou et al. (2013), to measure the implicit effects of the illusion, whilst the sizes of familiar objects were estimated by verbally stating whether the object looked larger or smaller than usual to measure the explicit effect of body size on size estimation. In Experiment 2, the effect of body size on size perception was assessed using a four-item questionnaire probing the subjective perception of body and environment sizes.

\section{Experiment 1: Effects of body size and visuomotor congruency on embodiment and size perception}

\section{Method}


Participants. Power analyses were carried out in $\mathrm{G}^{*}$ Power. Based on a predicted medium effect size of $f=.5$ and a desired power of .8 , the minimum total sample size was calculated to be 48 . Participants were 34 undergraduate students $\left(\mathrm{M}_{\mathrm{age}}=21.4\right.$ years, $\mathrm{SD}_{\mathrm{age}}=$ 2.0 years, 14 males $)$ and 34 children $\left(\mathrm{M}_{\mathrm{age}}=5.5\right.$ years, $\mathrm{SD}_{\mathrm{age}}=0.3$ years, 18 males $)$ living in the North-East of England. Participants were recruited from January 2018 to March 2019 and tested from February 2018 to April 2019. All participants had normal or corrected-to-normal vision and no motor impairments. This investigation was approved by the local research ethics committee. Informed consent was obtained from all adult participants, and the parents of all child participants included in the study.

Apparatus. All testing was carried out in a $5 \mathrm{~m}$ x 9m lab fitted with 16 Vicon Bonita cameras (Vicon, Oxford UK). This motion capture system tracks small, infrared reflective markers in real time at $240 \mathrm{~Hz}$, with millimetre accuracy. This allowed accurate tracking of live movement using 'clusters' of reflective markers attached to the arms, legs, and torso using Velcro straps (Figure 1a). Virtual bodies were created in MakeHuman (a free modelling software used to create 3D human avatars; www.makehumancommunity.org). The virtual body was mapped onto the participant's body using Vicon Pegasus software, such that the posture of the virtual and participant's bodies were matched. The virtual 'tea party' environment (Figure 1b) was created and implemented using Unity (Unity Technologies, San Francisco, CA, USA), and viewed by participants through an Oculus Rift head-mounted display (HMD; Oculus, Menlo Park, CA, USA).

Design. Virtual body size had three levels and was manipulated between-subjects. These were small, normal, and large; operationalised as $50 \%, 100 \%$, and $150 \%$ of 
participant's body size. As an index of body size we took body height, but the whole body was enlarged or reduced, not merely its height (Figure 1c-e). Proportions for both the small and large body conditions were determined in piloting to be sufficiently noticeable size changes. Visuomotor congruency had two levels (congruent, incongruent) and was manipulated within-subjects, with conditions counter-balanced to avoid order effects. Therefore, each participant experienced one virtual body size with both congruent and incongruent movements. We measured subjective embodiment using a questionnaire displayed within the VR environment. To measure the effect of body size on size estimation, we measured both manual size estimations of unfamiliar objects, and verbal size estimations of familiar objects. All measures are described in detail below.

Procedure. We used a procedure which has been established in literature describing embodiment in adults (Banakou et al., 2013) with seven steps, each described in detail below: i) Set-up, ii) Size estimation training, iii) Baseline size estimations, iv) Embodiment, v) Postembodiment size estimations, vi) Embodiment questionnaire, vii) Second embodiment phase, post-embodiment size estimations, and embodiment questionnaire.

i) Set-up

Before entering the virtual environment, each participant was shown example real objects to demonstrate the size estimation tasks. The first was a $30 \mathrm{~cm}$ white cube and the second was a familiar object (football), $22 \mathrm{~cm}$ in diameter. Participants were not told the metric size of the ball, but were instructed to remember its size, as they would need to recall it later. 

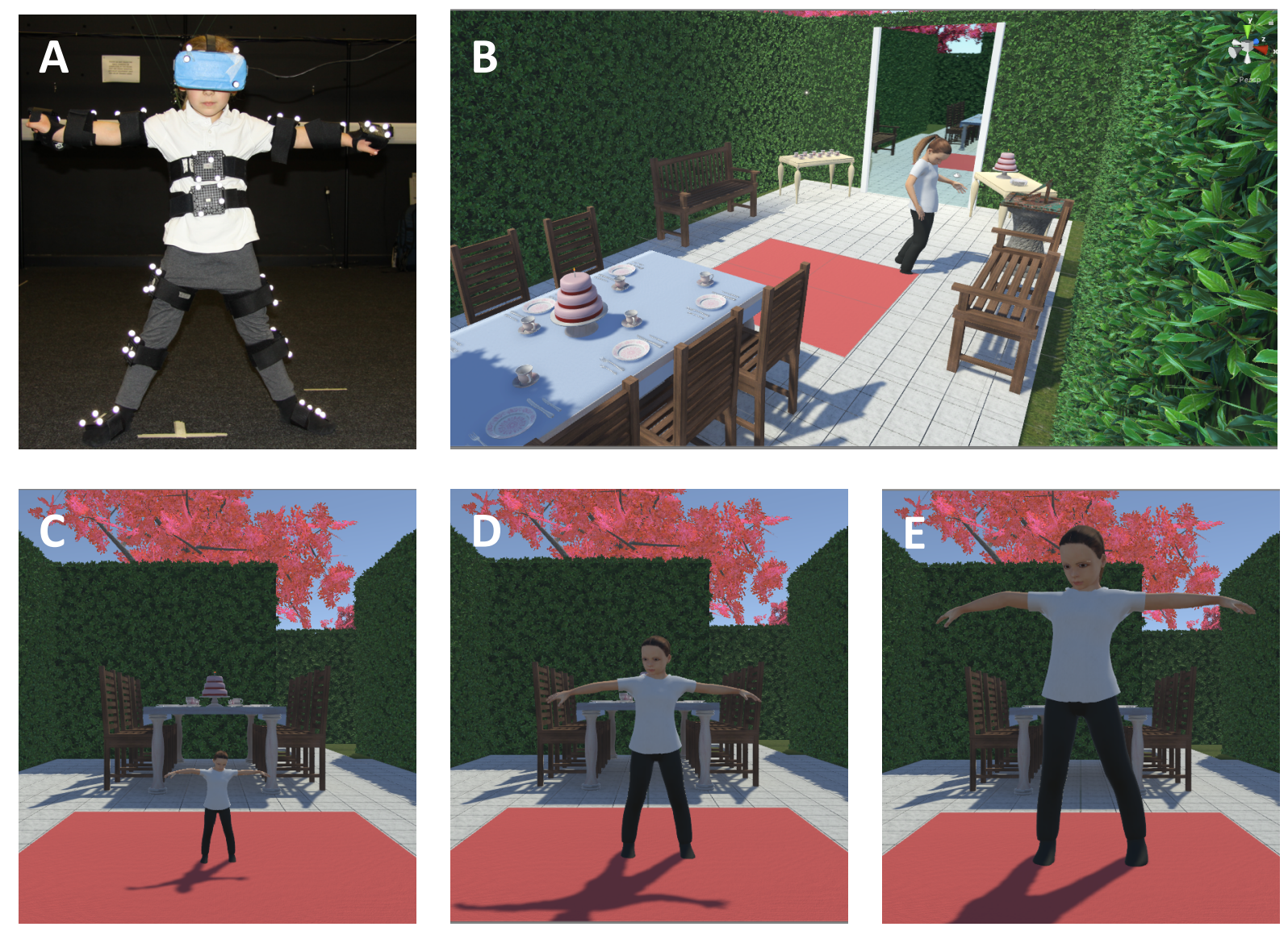

Figure 1. Methods. A Child participant wearing motion-capture clusters and the HMD. B The virtual 'tea party' scene with the child participant in the $\mathbf{C}$ Small body condition $\mathbf{D}$ Normal body condition $\mathbf{E}$ Large body condition.

Each participant's height was measured to the nearest centimetre, so that body size could be manipulated relative to the participant's actual height. They were then fitted with 'clusters' of reflective markers, used by motion capture cameras to track the participant's live movements, and an HMD, to allow participants to view a virtual body from a first-person perspective (Figure 1a). The experimenter taught participants a well-known, standardised set of full-body movements which therefore placed minimal demands on attention and memory (the children's dance 'the hokey cokey'), which they would carry out whilst inhabiting a virtual body. During the set-up phase, the experimenter took a motion capture recording of these movements lasting approximately two minutes for use in the incongruent motor condition. 
ii) Size estimation training

Participants carried out size estimation phases in a virtual outdoor scene resembling a large empty field. In this phase participants had no visible body, to prevent estimations being made based on visible hand position. The only objects in the scene were a scoreboard behind the participant indicating how many trials had been completed, and a white cube 'floated' at eye-level $30 \mathrm{~cm}$ away from the participant. Pilots showed that participants found the size estimations difficult in the absence of any cues to size or distance. Therefore, a plastic pole which could not be seen in VR was placed $30 \mathrm{~cm}$ in front of the participant. Participants could reach out to touch the pole, giving a distance cue which made size estimations easier. Cubes were one of three sizes: $15 \mathrm{~cm}, 25 \mathrm{~cm}$, and $35 \mathrm{~cm}$. Each cube size appeared three times in a random order for each participant.

For each cube, participants were instructed to hold their hands out in front of them to estimate the size of the cube, as if they were aiming to grasp it. The experimenter manually measured the distance between the participant's palms to the nearest centimetre (this could not easily be done through the software). Participants were given feedback with words appearing in VR reading 'TOO SMALL', 'CORRECT', or 'TOO BIG' (estimations were classified with $\pm 20 \%$ tolerance, so that for a $15 \mathrm{~cm}$ cube, estimates ranging from $12-18 \mathrm{~cm}$ were classed as correct). This feedback was also given verbally by the experimenter, e.g., "You guessed too big that time! Try a little smaller". Participants attempted the same cube until their estimation was rated as correct, at which point the next cube would appear.

After this, a virtual football the same size as the one presented in real life appeared at the same height and distance as the cubes. Participants were asked if the football was larger, smaller, or the same size as the real-life ball, and given feedback on their response.

iii) Baseline size estimations 
Baseline size estimations were carried out in the same outdoor environment as training, again with no visible virtual body. Participants saw 3 cubes $(10 \mathrm{~cm}, 20 \mathrm{~cm}, 30 \mathrm{~cm}) 3$ times each in a random order. Each was viewed at eye-level and at $30 \mathrm{~cm}$ distance, for 5 seconds before disappearing. Participants made their estimates, this time without feedback. Following this, another virtual football of the same size appeared and participants rated its size as before, but without feedback.

iv) Embodiment

In embodiment phases, participants were located in a virtual outdoor environment designed as a 'tea party'. Each participant was given an age-and gender-matched virtual body which was either $50 \%, 100 \%$, or $150 \%$ of their actual height. The body could be seen both by looking down from a first-person perspective and in a mirror located in front of the participant (Figure 1).

Participants were instructed to carry out the movements taught to them at the beginning of the procedure. In the congruent condition, the virtual body's movements were driven by the participant's own live movements. In the incongruent condition, the motion capture recording of the participant from the beginning of the experiment was used to move the virtual body incongruently to the participant's own movements, so that the felt and seen movements did not match. We achieved this by playing the recording from halfway through and then looping it, so that it played approximately one minute behind or in front the participant's live two-minute sequence. Importantly, participants did not try to synchronise their movements with that of the virtual body in the incongruent condition. Often, they were moving their upper limbs while the body moved its lower limbs, or vice versa. Visuomotor incongruency was therefore clearly perceptible to all participants. Participants were exposed to the virtual body for approximately two minutes in each embodiment phase. 
Table 1. Embodiment questionnaire statements.

\begin{tabular}{lll}
\hline Statement & Category & Label \\
\hline $\begin{array}{l}\text { While I was doing the hokey cokey, I felt as though the virtual } \\
\text { body I saw was my own body or belonged to me }\end{array}$ & Ownership & Own \\
\hline $\begin{array}{l}\text { While I was doing the hokey cokey, I felt like I was } \\
\text { controlling the movements of the virtual body }\end{array}$ & Agency & Agency \\
\hline While I was doing the hokey cokey I felt like I had a tail & Control & Tail \\
\hline $\begin{array}{l}\text { While I was doing the hokey cokey I felt like my hair was } \\
\text { turning blue }\end{array}$ & Control & Hair \\
\hline
\end{tabular}

v) Post-embodiment size estimations

Post-embodiment size estimations followed the same procedure as at baseline. In this way, we determined whether inhabiting the virtual body had an effect on the perceived size of surrounding objects.

vi) Embodiment questionnaire

Participants were shown a virtual outdoor environment, with a large blackboard presented in front of them. The blackboard showed one of four statements (Table 1) at a time. Participants indicated their agreement with each statement by moving a line on a continuous scale with their hand. The scale ranged from 'NO' ( $0 \%$ agreement) to 'YES' $(100 \%$ agreement). Of the four statements, one related to ownership of the virtual body, one related to agency over the virtual body's movements, and two were control questions. Statements were read aloud by the experimenter. The next statement appeared after the participant gave their response, in a randomised order for each participant. Before the first statement participants were trained to use the full range of the answer scale by asking them questions such as 'How much do you like chocolate? How much do you like mushrooms?'.

vii) Second embodiment phase, post-embodiment size estimations, and embodiment questionnaire 
Steps iv-vi were then repeated for the second visuomotor condition (congruent or incongruent depending on the order for that participant). Children received a small reward for taking part, and adults were awarded course credit if applicable.

\section{Results}

We carried out all analyses using IBM SPSS 22 or JASP. Bayes factor $\left(\mathrm{BF}_{10}\right)$ is reported for all parametric tests, indicating the likelihood of $\mathrm{H}_{1}$ compared to $\mathrm{H}_{0}$. In accordance with Kass and Raftery (1995), $\mathrm{BF}_{10}$ of 3.2 or lower is considered extremely weak evidence against $\mathrm{H}_{0}$, whilst $\mathrm{BF}_{10}$ of 10 or above is considered strong evidence against $\mathrm{H}_{0}$.

Data from six participants (three adults, three children) were excluded due to technical issues with the virtual environment, leaving data from 62 participants for analysis. Of these participants, 22 (11 adults, 11 children) experienced the normal body size condition, 20 (10 adults, 10 children) experienced the large body size condition, and 20 (10 adults, 10 children) experienced the small body condition.

Questionnaire. We examined the effects of visuomotor congruency and body size on agreement ratings using mixed ANOVA with between-subjects factors: age (child, adult) and body-size (small, normal, large) and within-subjects factors: congruency (congruent, incongruent), and questionnaire statement (hair, tail, ownership, agency). Questionnaire statement was included as a factor in order to identify differences in ownership and agency ratings. Where assumptions of sphericity were violated, Greenhouse-Geisser corrections were applied. A summary of the results is shown in Figure 2. 
Small
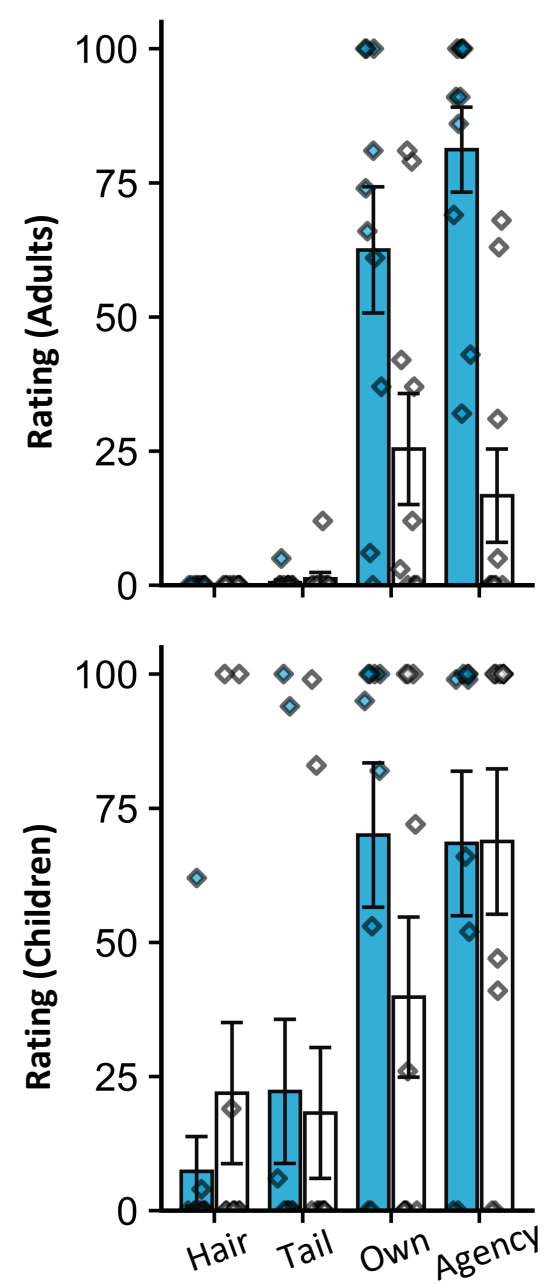

Visuomotor congruency
Normal
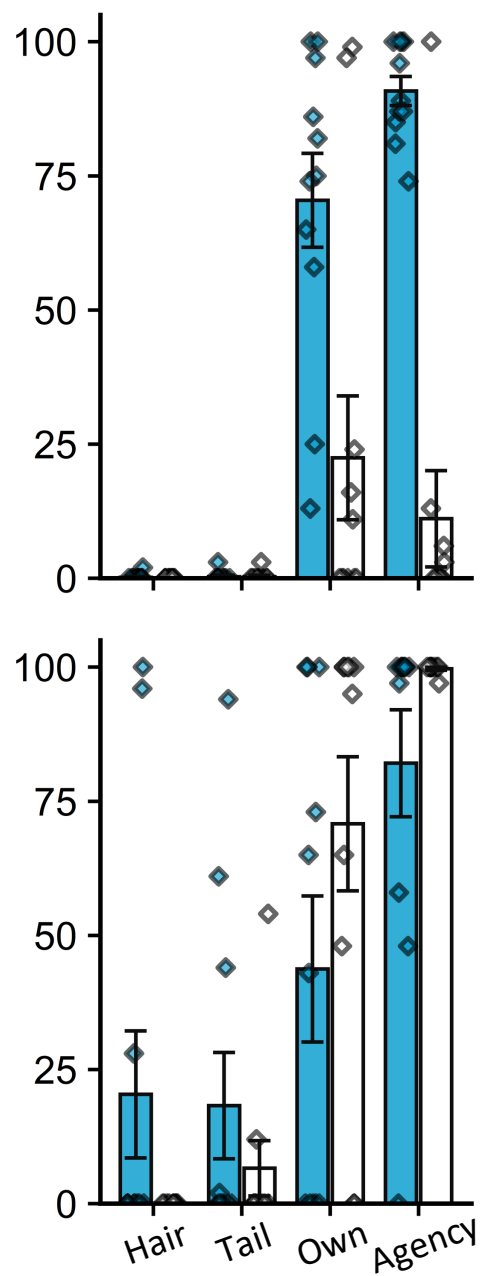

Statement
Large
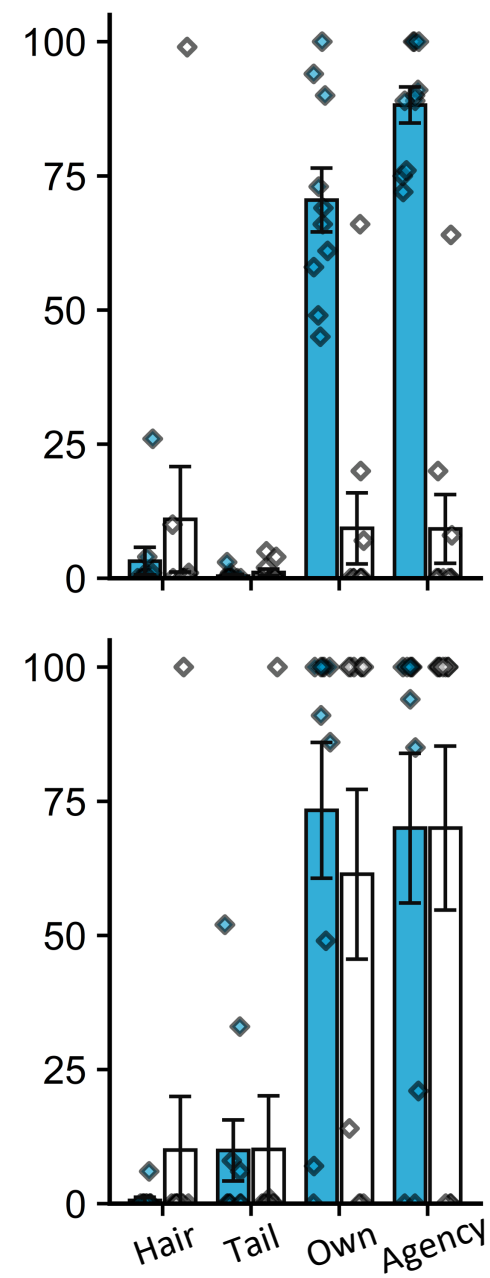

Figure 2. Experiment 1 Questionnaire Results. Mean (bar) and individual (scatter points) responses by virtual body size (small, normal, large), age group (children of 5 years; adults), and movement congruency (congruent, incongruent). Responses to control (hair, tail) and experimental (ownership, agency) questionnaire items (see Table 1 for details). Error bars represent standard error.

There was a significant effect of statement on agreement ratings $\left(F(2.28,122.85)=119.9, p<.001, \eta_{\mathrm{p}}^{2}=.689, \mathrm{BF}_{10}=4.608 \mathrm{e}+46\right)$. Pairwise comparisons using the Bonferroni correction revealed that ratings for the two control statements did not significantly differ $\left(p=1.0, \mathrm{BF}_{10}=.113\right)$, but were significantly lower than ratings for both embodiment 
questions $\left(p<.001, \mathrm{BF}_{10}>1.649 \mathrm{e}+15\right)$. Agency ratings were significantly higher than ownership ratings $\left(p=.032, \mathrm{BF}_{10}=9.66\right)$. Agreement ratings were further affected by visuomotor congruency $\left(F(1,54)=45.6, p<.001, \eta_{\mathrm{p}}{ }^{2}=.458, \mathrm{BF}_{10}=536.18\right)$, with ratings higher in the congruent condition than the incongruent condition; and age $(F(1,54)=23.0, p<.001$, $\eta_{\mathrm{p}}{ }^{2}=.298, \mathrm{BF}_{10}=28.34$ ), with children's mean ratings significantly higher than those of adults. However, body size did not significantly affect agreement ratings $(F(2,54)=0.2, p=.815$, $\left.\eta_{\mathrm{p}}^{2}=.008, \mathrm{BF}_{10}=.04\right)$.

There was a significant three-way interaction of congruency, questionnaire statement and age $\left(F(3,162)=18.44, p<.001, \eta_{\mathrm{p}}^{2} .208, \mathrm{BF}_{10}=9.691 \mathrm{e}+71\right)$. For adults, congruency did not affect agreement ratings for hair $\left(t(30)=-1.04, p=.307, \mathrm{BF}_{10}=.31\right)$ or tail $(t(30)=-1.73, p=.095$, $\left.\mathrm{BF}_{10}=.72\right)$ questionnaire statements. Adults' agreement ratings were significantly higher in the congruent condition than the incongruent condition for both ownership $(t(30)=8.46$, $\left.p<.001, \mathrm{BF}_{10}=6.037 \mathrm{e}+6\right)$ and agency $\left(t(30)=12.77, p<.001, \mathrm{BF}_{10}=6.228 \mathrm{e}+10\right)$ statements. However, children's agreement ratings were not affected by congruency for any of the four questionnaire statements (Hair: $t(28)=-.25, p=.806, \mathrm{BF}_{10}=.20$; Tail: $t(28)=.62, p=.544$, $\mathrm{BF}_{10}=.24$; Ownership: $t(28)=.48, p=.638, \mathrm{BF}_{10}=.22$; Agency: $t(28)=-.98, p=.337, \mathrm{BF}_{10}=.3$.

Size estimation. We examined size estimations of both unfamiliar objects (manual estimations of cube size) and familiar objects (verbal estimations of football size - 'smaller than', 'same as' or 'larger than' a real object). Two 'difference' variables were calculated for both unfamiliar and familiar object size estimations. These variables indicated the change in size estimation from baseline to post-embodiment in both congruent and incongruent conditions. 
Table 2. Mean differences in unfamiliar size estimation error $(\mathrm{cm})$ across conditions.

\begin{tabular}{lcccccc}
\hline & \multicolumn{9}{c}{ Body size \& Age } \\
\cline { 2 - 8 } & \multicolumn{2}{c}{ Small } & \multicolumn{2}{c}{ Normal } & \multicolumn{2}{c}{ Large } \\
\hline VM Congruency & Child & Adult & Child & Adult & Child & Adult \\
\hline Congruent & -2.2 & 1.4 & 1.8 & 1.7 & 0.5 & 0.8 \\
\hline Incongruent & 0.2 & 0.7 & 0.1 & 1.0 & 0.3 & 0.4 \\
\hline
\end{tabular}

We first examined differences in unfamiliar size estimations across conditions (Table 2) using mixed ANOVA with factors age (adult, child), body size (small, normal, large), and visuomotor congruency (congruent, incongruent). There were no significant effects of congruency $\left(F(1,55)=.19, p=.665, \eta_{\mathrm{p}}{ }^{2}=.003, \mathrm{BF}_{10}=.22\right)$, age $\left(F(1,55)=.50, p=.484, \eta_{\mathrm{p}}{ }^{2}=.009\right.$, $\left.\mathrm{BF}_{10}=.47\right)$ or body size $\left(F(2,55)=.27, p=.765, \eta_{\mathrm{p}}^{2}=.010, \mathrm{BF}_{10}=.30\right)$, and no significant interactions.

As the familiar object size estimation measure produced nominal-level data, we used Chi Squared tests to investigate any differences in this variable across conditions. We examined the relation between body size and familiar size estimation, separately for each age group and movement condition. There was no relation between body size and familiar size estimation in the incongruent condition (adults: $X^{2}(4)=.65, p=.957, \mathrm{BF}_{10}=3.7$; children: $\left.X^{2}(4)=5.13, p=.274, \mathrm{BF}_{10}=.16\right)$ or the congruent condition (adults: $X^{2}(4)=5.0, p=.288$, $\mathrm{BF}_{10}=.29$; children: $\left.X^{2}(4)=1.5, p=.823, \mathrm{BF}_{10}=.10\right)$.

\section{Experiment 1 Discussion}

Experiment 1 had three main findings. First, we replicated well-established adult findings regarding visuomotor cues to embodiment, i.e. adults accept a body that moves congruently with their own, but not one that moves incongruently (Kokkinara, Kilteni, Blom, \& Slater, 2016; Peck et al., 2013; Steptoe, Steed, \& Slater, 2013). However, we found that 5- 
year-olds reported high levels of ownership and agency over the virtual body regardless of visuomotor congruency. This may reflect a primacy of perspective over visuomotor congruency in young children's motor representations, allowing them to accept incongruent multisensory information when first-person perspective signals that the body is theirs. Since children did give very distinctively low ratings for control questions, we do not believe that this reflects a propensity for children to always agree with the statements given.

Secondly, we found that adults and children embodied virtual bodies of all three virtual body sizes, supporting previous adult work (Banakou et al., 2013; van der Hoort et al., 2011), and extending the findings to 5-year-olds. Since both adults and children were able to embody virtual bodies of different sizes to their own (both smaller and larger), it appears that neither age group holds strong top-down representations of body size. Anecdotally, children taking part in all conditions consistently referred to their virtual body as 'I' rather than using other third-person pronouns.

Lastly, we found no evidence of body size affecting size perception in either adults or children. This is in contrast to previous adult work, which has shown that illusory body size affects perception of the environment (Banakou et al., 2013; Tajadura-Jiménez, Banakou, Bianchi-Berthouze, \& Slater, 2017; van der Hoort et al., 2011). This result was unexpected as our method closely replicated that of Banakou et al. (2013). The main difference in our procedure was the inclusion of a tactile distance cue to help participants in their size estimations, which may have eliminated any effects of virtual body size. In Experiment 2 we further investigate the effect of body size on size estimation by asking participants about their subjective perceptual experience of their body and the environment. 


\section{Experiment 2: How body size affects body and environment size perception}

\section{Method}

Participants. Power analyses were carried out in $\mathrm{G}^{*}$ Power. Based on a predicted medium effect size of $f=.5$ and a desired power of .8 , the total required sample size was calculated to be 24 . Participants were 12 undergraduate students $\left(\mathrm{M}_{\mathrm{age}}=19.9\right.$ years, $\mathrm{SD}_{\mathrm{age}}=$ 1.3 years, 10 female) and 125 -year-old children $\left(\mathrm{M}_{\text {age }}=5.4\right.$ years, $\mathrm{SD}_{\text {age }}=0.3$ years, 2 female) recruited from the North-East of England. Participants were recruited from November 2018 to March 2019 and tested between December 2018 and April 2019. All participants had normal or corrected-to-normal vision and no motor impairments. This investigation was approved by the local research ethics committee. Informed consent was obtained from all adult participants, and the parents of all child participants included in the study.

Apparatus. Apparatus was the same as in Experiment 1.

Design. In Experiment 2, virtual body size had two levels (small, large; operationalised as $50 \%$ and $150 \%$ of participant's body height respectively), and was manipulated within-subjects. The order in which body size conditions were presented was counter-balanced to avoid order effects. Subjective size estimations were measured using a virtual questionnaire with a similar structure to the embodiment questionnaire in Experiment 1. In Experiment 2, the virtual body always moved congruently with the participant's own.

Procedure. As in Experiment 1, participants' height was measured to the nearest centimetre in order to accurately manipulate virtual body size. Participants were then fitted 
Table 3. Size questionnaire statements.

\begin{tabular}{ll}
\hline Statement & Category \\
\hline $\begin{array}{l}\text { When I was at the tea party it felt as though the body I saw was smaller } \\
\text { than my own body }\end{array}$ & Body smaller \\
\hline $\begin{array}{l}\text { When I was at the tea party it felt as though the body I saw was bigger } \\
\text { than my own body }\end{array}$ & Body bigger \\
\hline $\begin{array}{l}\text { When I was at the tea party it felt as though objects around me were } \\
\text { smaller than normal }\end{array}$ & Objects smaller \\
\hline $\begin{array}{l}\text { When I was at the tea party it felt as though objects around me were } \\
\text { bigger than normal }\end{array}$ & Objects bigger \\
\hline
\end{tabular}

with clusters of motion-tracking markers and taught the same set of movements as in Experiment 1 (the 'hokey cokey').

Participants were immersed in the same 'tea party' virtual environment with a mirror. In each body size condition participants performed the movements taught to them by the experimenter, which lasted approximately two minutes. After this phase, participants were shown a large blackboard which sequentially showed one of four statements in a random order (statements are shown in Table 3). Participants rated their agreement with each statement in the same manner as in Experiment 1. These steps were then repeated for the second body size condition.

\section{Results}

For Experiment 2 we were interested in agreement ratings for each questionnaire statement but not their comparison. Therefore, ratings were examined for each statement separately using four mixed ANOVAs, with each having within-subjects factor body size (small, large) and between-subjects factor age (adult, child). Ratings for each question are shown in Figure 3. 
Body felt smaller

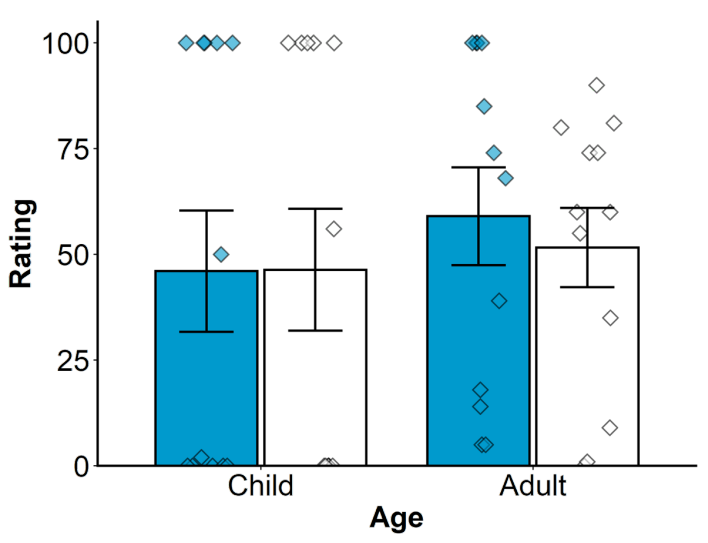

Objects felt smaller

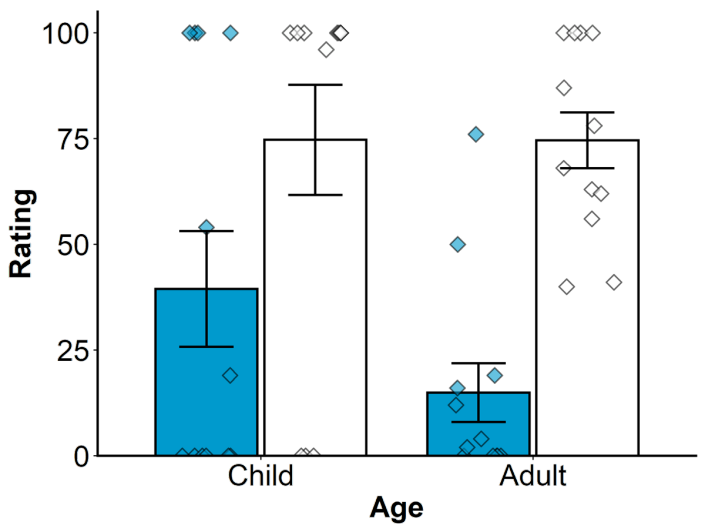

Body felt larger

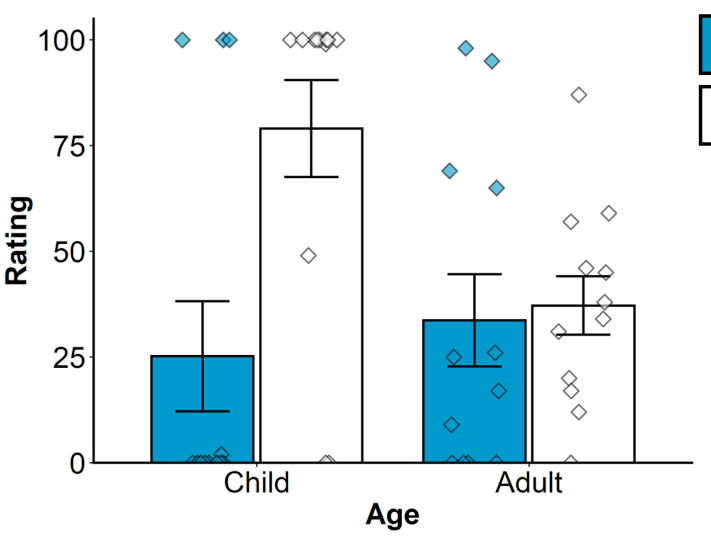

Objects felt larger

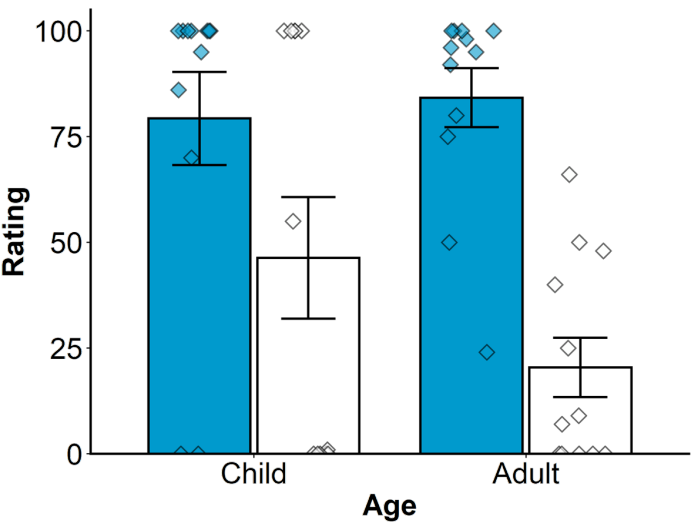

Figure 3. Experiment 2 Questionnaire Results. Mean (bars) and individual (scatter points) ratings for questionnaire items a) 'Body smaller'; b) 'Body larger'; c) 'Object smaller'; d) 'Object larger' (see Table 3 for details). Shown by age (adult, child) and virtual body size (large, small). Movement was always congruent. Errors represent standard error.

For the 'body smaller' question, there was no effect of body size $(F(1,22)=.08$, $\left.p=.787, \eta_{\mathrm{p}}{ }^{2}=.003, \mathrm{BF}_{10}=.30\right)$ or age $\left(F(1,22)=.55, p=.464, \eta_{\mathrm{p}}{ }^{2}=.025, \mathrm{BF}_{10}=.41\right)$ on agreement ratings, and no interaction $\left(F(1,22)=.09, p=.767, \eta_{\mathrm{p}}^{2}=.004, \mathrm{BF}_{10}=.05\right)$.

'Body larger' agreement ratings were significantly affected by body size $\left(F(1,22)=9.55, p=.005, \eta_{\mathrm{p}}{ }^{2}=.303, \mathrm{BF}_{10}=7.79\right)$ with ratings higher in the large body condition than the small body condition. Ratings were not significantly affected by age $(F(1,22)=1.88$, $\left.p=.184, \eta_{\mathrm{p}}{ }^{2}=.079, \mathrm{BF}_{10}=.60\right)$, but there was a significant interaction of body size and age $\left(F(1,22)=7.36, p=.013, \eta_{\mathrm{p}}^{2}=.251, \mathrm{BF}_{10}=30.07\right)$. Paired t-tests reveal that children rated 'body 
larger' higher in the large body condition than the small body condition $(t(1)=3.73, p=.003$, $\left.\mathrm{BF}_{10}=14.72\right)$, whereas adults did not $\left(t(11)=.30, p=.770, \mathrm{BF}_{10}=.30\right)$.

'Object smaller' ratings were significantly higher in the large body condition than the small body condition $\left(F(1,22)=23.29, p<.001, \eta_{\mathrm{p}}^{2}=.514, \mathrm{BF}_{10}=2194.41\right)$, but were not significantly affected by age $\left(F(1,22)=1.19, p=.287, \eta_{\mathrm{p}}{ }^{2}=.051, \mathrm{BF}_{10}=.44\right)$. There was no significant interaction of body size and age for this question according to the p-value, though Bayes factor indicated some evidence of an interaction $\left(F(1,22)=1.54, p=.228, \eta_{\mathrm{p}}{ }^{2}=.065\right.$, $\left.\mathrm{BF}_{10}=809.23\right)$. Investigating this further, adults' ratings were significantly higher in the large body condition than the small body condition $\left(p<.001, \mathrm{BF}_{10}=136.50\right)$, whereas this difference was marginal for children $\left(p=.052, \mathrm{BF}_{10}=1.61\right)$.

Finally, 'object larger' agreement ratings were also significantly affected by body size $\left(F(1,22)=20.17, p<.001, \eta_{\mathrm{p}}{ }^{2}=.478, \mathrm{BF}_{10}=1976.56\right)$, with ratings higher in the small body condition than the large body condition. There was no significant effect of age on this question $\left(F(1,22)=1.14, p=.297, \eta_{\mathrm{p}}{ }^{2}=.049, \mathrm{BF}_{10}=.42\right)$. Again, although the $\mathrm{p}$-value indicated that there was no significant interaction, Bayes factor suggested otherwise $(F(1,22)=2.05$, $\left.p=.166, \eta_{\mathrm{p}}^{2}=.085, \mathrm{BF}_{10}=850.59\right)$. Again, adults rated this question higher in the small body condition, and the difference was marginal in children $\left(p=.095, \mathrm{BF}_{10}=1.03\right)$.

\section{Experiment 2 Discussion}

In Experiment 2, we aimed to better understand the perceptual experience of participants when inhabiting a virtual body that was a different size to their own. Specifically, do adults and children perceive their body to have changed size, or rather their environment? Here we found that in the small body condition, neither adults nor children reported a change in perceived body size - rather, both reported that objects in the environment appeared larger 
(though this effect was somewhat weaker in children than in adults). However, in the large body condition, adults did not report a change in perceived body size, whereas children did report perceiving their body as larger. Both age groups also reported that objects in the environment seemed smaller than usual (though again, this was slightly more apparent in adults than children).

In summary, when presented with a virtual environment where the relative sizes of body and environment are different to usual, both adults and children primarily report that objects in the environment change size whilst their own body size remains stable. The notable exception is that children in the large body size condition do report their body as bigger than usual. It may be that 5-year-old children are accustomed to their body growing and so are more willing to accept a body size change in this direction. In contrast, neither adults nor children reported their body as smaller in the small body condition, potentially because our bodies do not generally shrink at any age.

\section{General Discussion}

We have examined, for the first time, the effect of full-body visuomotor congruency on own-body representation in young children, as well as the effect of body size on their own-body representation and size perception. We found that, whilst adults showed higher levels of embodiment over a virtual body which moved congruently with their own than one which moved incongruently, children embodied an avatar regardless of visuomotor congruency. It was also found that both adults and 5-year-old children embodied, to an equal extent, virtual bodies that were smaller, larger, or the same size as their own. Finally, we found that both adults and children reported that objects in the environment were smaller after experiencing a large body, and larger after experiencing a small body. Whilst adults did not report changes in body size in either condition, children did report that their body was 
larger after the large body condition. These findings and their implications are discussed in more detail below.

\section{The role of visuomotor congruency in embodiment}

Adults gave higher embodiment ratings when a virtual body moved congruently with their own movements than when felt and seen movements were incongruent. These results are in accordance with the well-replicated finding that adults rely on the congruency of multisensory cues for own-body representation (Kokkinara et al., 2016; Peck et al., 2013; Steptoe et al., 2013), and therefore provide further support to this idea. Replicating this result in adults provides assurance that the experimental manipulation of visuomotor congruency worked as expected, allowing us to place confidence in the results of the younger group.

As hypothesised, children's embodiment ratings were not affected by visuomotor congruency, as supported by p-values and Bayes factors. Remarkably, children embodied an avatar even when it moved completely randomly compared to their own movements, often moving entirely different limbs to the participant. It is important to note that these questions are comprehensible to children and have previously produced differential responses from children of this age for changes in e.g. visuotactile synchrony (Cowie et al., 2013, 2018, 2016) or hand posture (Gottwald et al., 2021). We took care in training to have children use the full range of the answer scale and they successfully did this before testing commenced. Further, in the test session children's ratings on the critical embodiment questions were always significantly higher than their ratings of control questions. We can therefore rule out the possibility that children responded positively to all questions. Likewise, it is also unlikely that the results were caused by a lack of sensitivity to the visuomotor discrepancy in the incongruent condition. Whilst young children do show a wider temporal binding window for multisensory cues than older children and adults, this is still of the order of hundreds of 
milliseconds (Lewkowicz \& Flom, 2014) rather than the $\sim$ one minute discrepancy between proprioceptive and visual cues in the present study. It is therefore more likely that the 5-yearolds did notice the discrepancy but that this did not diminish their feelings of embodiment.

This result suggests for the first time that, at 5 years, children do not substantially use the congruency of visuomotor cues in embodying a full body, when strong perspective cues to body ownership are present. Indeed, in judging whether a viewed body is their own, they discounted large, perceptible visuomotor discrepancies to conclude that the viewed body was their own and that they could control its movement. This contrasts with recent work showing that movement synchrony affects ownership over a virtual hand in mid-childhood (Dewe et al., 2021) and over a virtual body at 8-12 years (Weijs et al., 2021). However, we note that our participants were substantially younger than in these studies, and that we have previously found development from 6-11 years in full-body embodiment (Cowie et al., 2018). Further, in one of these studies even at 8 years children viewing 1-second-delayed movements did show strong threat responses, as well persistently high agency compared to adults (Weijs et al., 2021). Finally, the result is in line with previous research showing that perspective is a very strong cue to own-body representation in adults: merely viewing a body from a first-person perspective can be sufficient to induce a sense of embodiment (Keenaghan et al., 2020; Kokkinara et al., 2016; Slater, Spanlang, Sanchez-Vives, \& Blanke, 2010).

Further work is needed which uses the same paradigm to measure own-body representation across a wide age range of children. This should give a fuller picture of the developmental trajectory of how children weight perspective and movement cues for embodiment; how children might use synchrony in finer-grained ways or when perspective cues point less strongly to ownership; how synchrony might affect aspects of embodiment not measured here (e.g. affective response); how specific embodiment is to one's own body form, 
or to biological motion; and how explicit embodiment responses are linked to developing perceptual sensitivities to multisensory information.

\section{The role of body size in embodiment}

We found that the size of a virtual avatar did not alter adults' ratings of embodiment. This supports and extends previous findings that adults embody smaller or larger bodies than their own in congruent multisensory conditions (Banakou et al., 2013; van der Hoort et al., 2011; van der Hoort \& Ehrsson, 2014). We can now conclude that, not only do adults accept bodies of different sizes, but also that they accept these bodies to an equal extent.

The present study also provided the first investigation into the effect of body size on own-body representation in children. Based on the premise that children's bodies are naturally and rapidly changing size during development, it was hypothesised that children would show a similar effect to adults and embody an avatar regardless of body size. The present results support this hypothesis: embodiment ratings did not differ across body size conditions for either age group. One possibility is that rather than feeling their body had changed size, participants felt that it had moved in depth relative to the mirror. However, this would contradict motor cues indicating a stationary body as well as the results from Experiment 2 indicating that surrounding objects had changed size; and we received no reports of perceived depth or position changes. We conclude that both children and adults perceived differently-sized bodies to be their own, basing their judgment on the viewed avatar body rather than any internal representations of body size.

This finding demonstrates considerable flexibility in body representation for both adults and children, in accordance with the whole-body literature. Adults hold certain topdown representations of body form, meaning that embodiment is restricted by an object's appearance as 'body-like'; for example, adults do not embody objects such a wooden blocks 
or sticks (Lenggenhager et al., 2007; Tsakiris \& Haggard, 2005). In contrast, the fact that both adults and children embody avatars of different sizes suggests that own-body representation is not restricted by internal representations of body size. Indeed, van der Hoort et al. (2011) speculate that the range of body sizes which adults are able to embody may be unlimited, as long as the body remains in proportion (i.e. basic body form remains the same). The present findings support this view and extend its scope to children as well as adults. Alongside other recent work (Hoyet, Argelaguet, Nicole, \& Lécuyer, 2016), the data also fail to support the 'rubber band hypothesis' (de Vignemont, 2018), which states that the body map may be stretched but not compressed. Future investigations should aim to identify if children also hold similar representations of body form as adults, or whether they are more adaptable regarding the forms that they can inhabit.

\section{Size perception in differently sized avatars}

In Experiment 1, neither children nor adults showed any effect of body size on size estimations of either unfamiliar or familiar objects. Though this effect had not previously been investigated in children, our findings are inconsistent with previous work in adults. Previous findings have shown that inducing illusory ownership over a smaller or larger body than one's own led to an increase or decrease respectively in size estimations of objects in the environment (Banakou et al., 2013; van der Hoort et al., 2011), which is referred to as bodyrelative scaling (Linkenauger et al., 2010). Our result was unusual, as the methods were a partial replication of those used by (Banakou et al., 2013). However, we did include a touch reference, allowing participant to feel how far away the virtual objects were. This was introduced during piloting, as without it participants were unable to give consistent and accurate size estimations, even with extensive training. This may have reduced uncertainty to an extent where body size no longer affected size estimations. Indeed, Chen, Sperandio, and 
Goodale (2018) found that unseen tactile cues to distance provided a sense of size constancy in the absence of other cues.

In Experiment 2, however, questions showed that both children and adults demonstrated evidence of body-relative scaling, as the environment was perceived to be smaller in the large body condition and vice versa for both groups. These results support the previous findings on size perception from implicit measures like size estimation in adults (Banakou et al., 2013; Linkenauger et al., 2010; van der Hoort et al., 2011). Importantly, this was also the first study to have examined the effect of body size on size perception in children. This question was of theoretical interest as, of course, children's bodies are continuously changing size, as opposed to adult bodies whose sizes stay mostly constant. This could feasibly mean that children would not use their body size as a reference due its lack of reliability. However, we did not find this to be the case. The 5-year-old group responded in much the same manner as the adults, suggesting that they do in fact use body size as a cue to size perception in the environment. However, notably, in the large body condition children did report their body to have grown where adults did not. We suggest that this could be due to young children being more 'tuned-in' to their body growth, as this is something they must account for as they develop, therefore they may be more likely than adults to notice such a change. Further work should test a broader age range in order to confirm whether this effect does diminish as body growth slows. This finding was also complemented by the fact that neither adults nor children reported their body to have shrunk in the small body condition. Participants may be less sensitive to changes in body size in this direction as it does not normally occur naturally during the lifespan.

\section{Practical implications}


Overall, the present findings demonstrate that the full-body illusion can be elicited in children as young as 5 years old, and that children embody avatars regardless of visuomotor congruency and body size. We have also shown that both 5-year-olds and adults are susceptible to body-relative scaling, whereby their perception of the size of their environment is affected by the size of their embodied avatar. It is important to note that our sample was drawn from a predominantly WEIRD population (Rad, Martingano, \& Ginges, 2018), as the North-East of England comprises 92\% white-British populates, according to the British Office for National Statistics, 2009. Therefore, we must be conscious of avoiding overgeneralisation of the results. However, our findings add a great deal to our theoretical understanding of the development of own-body representation. Practically, we have also confirmed that full-body motion tracking and immersive virtual reality can be used successfully in young children (see also Weijs et al, 2020). Such technology is being widely used in adults to investigate social interactions (e.g. Peck et al., 2013), and may be useful in physical rehabilitation (Levin, Weiss, \& Keshner, 2015). Based on the present findings, it is plausible that these important applications could now be used with children as young as 5 years old.

\section{Conclusion}

The findings of the present study make a valuable contribution to current understanding of own-body representation and its development. We show that children embody a moving avatar regardless of its visuomotor congruency, highlighting both the flexibility of motor representations in young children, and the potential importance of perspective in children's body representation. We have also shown that both adults and children can embody avatars of different sizes, supporting and extending previous findings that body representation is not restricted by representations of body size (e.g. van der Hoort et al., 2011). Though we did not replicate previous findings that body size affects implicit size perception, we did find that 
both adults and children explicitly report the environment to appear smaller when they

inhabit a large body, and vice versa, with children also reporting a change in body size in the large body condition. Therefore, we have provided evidence of body-relative scaling in 5year-olds. Though much is still unknown about the development of own-body representation (e.g. the effect of perspective and importance of body form), the present study provides an essential starting point for future investigations.

Acknowledgements. We thank the children and adults who participated in this study. The data that support the findings of this study are openly available in OSF at https://doi.org/10.31234/osf.io/uw768. 


\section{References}

Bahrick, L. E., \& Watson, J. S. (1985). Detection of intermodal proprioceptive-visual contingency as a potential basis of self-perception in infancy. Developmental Psychology, 21(6), 963-973. https://doi.org/10.1037/0012-1649.21.6.963

Banakou, D., Groten, R., \& Slater, M. (2013). Illusory ownership of a virtual child body causes overestimation of object sizes and implicit attitude changes. Proceedings of the National Academy of Sciences, 110(31), 12846-12851.

https://doi.org/10.1073/pnas.1306779110

Bermúdez, J. L. (2005). The phenomenology of bodily awareness. In D. Woodruff Smith \& A. L. Thomasson (Eds.), Phenomenology and Philosophy of Mind (pp. 295-316). New York: Oxford University Press.

Bischof-Köhler, D. (2012). Empathy and self-recognition in phylogenetic and ontogenetic perspective. Emotion Review, 4(1), 40-48. https://doi.org/10.1177/1754073911421377

Blanke, O., \& Metzinger, T. (2009). Full-body illusions and minimal phenomenal selfhood. Trends in Cognitive Sciences, 13(1), 7-13. https://doi.org/10.1016/j.tics.2008.10.003

Botvinick, M., \& Cohen, J. (1998). Rubber hands "feel" the touch that eyes see. Nature, 391(6669), 756.

Brooks-Gunn, J., \& Lewis, M. (1984). The development of early visual self-recognition. Developmental Review, 4, 215-239.

Brownell, C. A., Zerwas, S., \& Ramani, G. B. (2007). "so big”: The development of body self-awareness in toddlers. Child Development, 78(5), 1426-1440. https://doi.org/10.1111/j.1467-8624.2007.01075.x

Chen, J., Sperandio, I., \& Goodale, M. (2018). Proprioceptive distance cues restore perfect 
size constancy in grasping, but not perception, when vision is limited. Current Biology, 28(6), 927-932.

Cowie, D., Makin, T. R., \& Bremner, A. J. (2013). Children's responses to the rubber-hand illusion reveal dissociable pathways in body representation. Psychological Science, 24(5), 762-769. https://doi.org/10.1177/0956797612462902

Cowie, D., McKenna, A., Bremner, A. J., \& Aspell, J. E. (2018). The development of bodily self-consciousness: changing responses to the Full Body Illusion in childhood. Developmental Science, 21(3), 1-12. https://doi.org/10.1111/desc.12557

Cowie, D., Sterling, S., \& Bremner, A. J. (2016). The development of multisensory body representation and awareness continues to 10years of age: Evidence from the rubber hand illusion. Journal of Experimental Child Psychology, 142, 230-238. https://doi.org/10.1016/j.jecp.2015.10.003

De Klerk, C. C. J. M., Filippetti, M. L., \& Rigato, S. (2021). The development of body representations: An associative learning account. Proceedings of the Royal Society B: Biological Sciences, 288(1949). https://doi.org/10.1098/rspb.2021.0070

de Vignemont, F. (2018). Mind the Body. New York: Oxford University Press.

DeLoache, J. S., Uttal, D. H., \& Rosengren, K. S. (2004). Scale errors offer evidence for a perception-action dissociation early in life. Science, 304(5673), 1027-1029.

Dewe, H., Gottwald, J., Bird., L., Brenton, H., Gillies, M., \& Cowie, D. (2021). My virtual self: the role of movement in children's sense of embodiment. IEEE Transactions on Visualization and Computer Graphics. https://doi.org/10.1109/TVCG.2021.3073906

Fausey, C. M., Jayaraman, S., \& Smith, L. B. (2016). From faces to hands: changing visual input in the first two years. Cognition, 152, 101-107. 
Filippetti, M L, Johnson, M. H., Lloyd-Fox, S., Dragovic, D., \& Farroni, T. (2013). Body perception in newborns. Current Biology, 23(23), 2413-2416. https://doi.org/10.1016/j.cub.2013.10.017

Filippetti, Maria Laura, \& Crucianelli, L. (2019). If I were a grown-up: Children's response to the rubber hand illusion with different hand sizes. Journal of Experimental Child Psychology, 185, 191-205. https://doi.org/10.1016/j.jecp.2019.04.016

Gottwald, J. M., Bird, L. A., Keenaghan, S., Diamond, C., Zampieri, E., Tosodduk, H., ... Cowie, D. (2021). The Developing Bodily Self: How Posture Constrains Body Representation in Childhood. Child Development, 92(1), 351-366. https://doi.org/10.1111/cdev.13425

Greenfield, K., Ropar, D., Smith, A. D., Carey, M., \& Newport, R. (2015). Visuo-tactile integration in autism: Atypical temporal binding may underlie greater reliance on proprioceptive information. Molecular Autism, 6(1), 1-10. https://doi.org/10.1186/s13229-015-0045-9

Hamilton, D., McKechnie, J., Edgerton, E., \& Wilson, C. (2021). Immersive virtual reality as a pedagogical tool in education: a systematic literature review of quantitative learning outcomes and experimental design. In Journal of Computers in Education (Vol. 8). https://doi.org/10.1007/s40692-020-00169-2

Hoyet, L., Argelaguet, F., Nicole, C., \& Lécuyer, A. (2016). "Wow! i have six fingers!": Would you accept structural changes of your hand in VR? Frontiers Robotics AI, 3(MAY), 1-12. https://doi.org/10.3389/frobt.2016.00027

Kass, R. E., \& Raftery, A. E. (1995). Bayes factors. Journal of the American Statistical Association, 90(430), 773-795. 
Keenaghan, S., Bowles, L., Crawfurd, G., Thurlbeck, S., Kentridge, R. W., \& Cowie, D. (2020). My body until proven otherwise: Exploring the time course of the full body illusion. Consciousness and Cognition, 78(January), 102882. https://doi.org/10.1016/j.concog.2020.102882

Kokkinara, E., Kilteni, K., Blom, K. J., \& Slater, M. (2016). First person perspective of seated participants over a walking virtual body leads to illusory agency over the walking. Scientific Reports, 6(28879). https://doi.org/10.1038/srep28879

Lenggenhager, B., Tadi, T., Metzinger, T., \& Blanke, O. (2007). Video ergo sum: Manipulating bodily self-consciousness. Science, 317(5841), 1096-1099. https://doi.org/10.1126/science.1144876

Levin, M. F., Weiss, P. L., \& Keshner, E. A. (2015). Emergence of virtual reality as a tool for upper limb rehabilitation: Incorporation of motor control and motor learning Principles. Physical Therapy, 95(3), 415-425. https://doi.org/10.2522/ptj.20130579

Lewis, M., \& Ramsay, D. (2004). Development of self-recognition, personal pronoun use, and pretend play during the 2nd Year. Child Development, 75(6), 1821-1831. https://doi.org/10.2307/3696679

Lewkowicz, D. J., \& Flom, R. (2014). The audio-visual temporal binding window narrows in early childhood. Child Development, 85(2), 685-694.

Linkenauger, S. A., Ramenzoni, V., \& Proffitt, D. R. (2010). Illusory shrinkage and growth: Body-based rescaling affects the perception of size. Psychological Science, 21(9), 13181325. https://doi.org/10.1177/0956797610380700

Marino, B. F., Stucchi, N., Nava, E., Haggard, P., \& Maravita, A. (2010). Distorting the visual size of the hand affects hand pre-shaping during grasping. Experimental Brain 
Research, 202, 499-505.

Negen, J., Heywood-Everett, E., Roome, H. E., \& Nardini, M. (2017). Developmemt of allocentric spatiall recall from new viewpoints in virtual reality. Developmental Science, 21(1), e12496. https://doi.org/https://doi.org/10.1111/desc.12496

Newport, R., Auty, K., Carey, M., Greenfield, K., Howard, E. M., Ratcliffe, N., ... Themelis, K. (2015). Give it a tug and feel it grow: Extending body perception through the universal nature of illusory finger stretching. I-Perception, 6(5), 1-4.

Pavani, F., \& Zampini, M. (2007). The role of hand size in the fake-hand illusion. Perception, $36,1547-1554$.

Peck, T. C., Seinfeld, S., Aglioti, S. M., \& Slater, M. (2013). Putting yourself in the skin of a black avatar reduces implicit racial bias. Consciousness and Cognition, 22(3), 779-787. https://doi.org/10.1016/j.concog.2013.04.016

Rad, M. S., Martingano, A. J., \& Ginges, J. (2018). Toward a psychology of Homo sapiens: Making psychological science more representative of the human population. PNAS, $115(45), 11401-11405$.

Slater, M., Spanlang, B., Sanchez-Vives, M. V, \& Blanke, O. (2010). First person experience of body transfer in virtual reality. PLoS One, 5(5), e10564.

Steptoe, W., Steed, A., \& Slater, M. (2013). Human tails: Ownership and control of extended humanoid avatars. IEEE Transactions on Visualization and Computer Graphics, 19(4), 583-590. https://doi.org/10.1109/TVCG.2013.32

Tajadura-Jiménez, A., Banakou, D., Bianchi-Berthouze, N., \& Slater, M. (2017).

Embodiment in a child-like talking virtual body influences object size perception, selfidentification, and subsequent real speaking. Scientific Reports, 7(9637). 
https://doi.org/10.1038/s41598-017-09497-3

Tsakiris, M., \& Haggard, P. (2005). The rubber hand illusion revisited: Visuotactile integration and self-attribution. Journal of Experimental Psychology: Human Perception and Performance, 31(1), 80-91. https://doi.org/10.1037/0096-1523.31.1.80

van der Hoort, B., \& Ehrsson, H. H. (2014). Body ownership affects visual perception of object size by rescaling the visual representation of external space. Attention, Perception, \& Psychophysics, 76(5), 1414-1428. https://doi.org/10.3758/s13414-0140664-9

van der Hoort, B., Guterstam, A., \& Ehrsson, H. H. (2011). Being barbie: The size of one's own body determines the perceived size of the world. PLOS ONE, 6(5). https://doi.org/10.1371/journal.pone.0020195

Weijs, M. L., Macartney, E., Daum, M. M., \& Lenggenhager, B. (2021). Development of the bodily self: Effects of visuomotor synchrony and visual appearance on virtual embodiment in children and adults. Journal of Experimental Child Psychology, 210, 105200. https://doi.org/10.1016/j.jecp.2021.105200

Won, A., Bailey, J., Bailenson, J., Tataru, C., Yoon, I., \& Golianu, B. (2017). Immersive Virtual Reality for Pediatric Pain. Children, 4(7), 52. https://doi.org/10.3390/children4070052 